\title{
Radiothérapie et cancer du sein : le point sur les outils développés en recherche translationnelle
}

\author{
Radiotherapy and Breast Cancer : Update on the Tools developed in Translational Research
}

\author{
C. Bourgier \\ (C) Lavoisier SAS 2017
}

À l'ère de la médecine personnalisée, de nombreux outils ont été développés afin de déterminer le pronostic individuel des patientes et d'optimiser les stratégies thérapeutiques. Ainsi, les signatures moléculaires ont permis d'identifier des soustypes de cancers du sein, de mieux appréhender le pronostic de ces patientes et d'adapter la stratégie thérapeutique.

Concernant le contrôle locorégional, B. Bataille, L. Boulanger, N. Kotecki, et al. [1] soulignent que le risque de récidive local semble être augmenté dans les phénotypes de type Her2 et basal-like. Toutefois, les signatures moléculaires ne sont pas à ce jour utilisées prospectivement en radiothérapie. Les données sont plus matures concernant les traitements systémiques et ces signatures sont en train de modifier la prise en charge adjuvante. L'article de N. Joyon, F. Penault-Llorca et M. Lacroix-Triki est une mise au point sur cette thématique [2].

Outre l'utilisation des signatures moléculaires, d'autres technologies et modalités de prélèvements telles que les biopsies liquides ont été développées dans le cadre de la surveillance du devenir de nos patientes, de l'évaluation de l'efficacité des traitements, en particulier les cellules tumorales circulantes (CTC). Ces dernières apportent des informations complémentaires aux biopsies conventionnelles, notamment en termes d'informations moléculaires et phénotypiques. Outre le caractère pronostique des CTC après radiothérapie de nombreuses tumeurs primitives, des données de la littérature suggèrent que la radiothérapie induit la mobilisation des CTC (cancers du sein, bronchique non à petites cellules, VADS) et posent la question d'un possible réensemencement. Ces sujets seront abordés dans l'article proposé par C. Alix de Panabières et J.Y. Pierga [3].

Enfin, l'interaction entre radiothérapie et cellules souches tumorales apporte également de nouvelles connaissances sur les mécanismes de radiorésistance, notamment via le processus de reprogrammation cellulaire. De nouvelles stratégies thérapeutiques émergent dans ce domaine et sont détaillées dans l'article de J. Bailleul-Dubois, N. Bidan, X. Le Bourhis et C. Lagadec [4].

Ce dossier thématique vous propose une mise à jour sur les outils développés en recherche translationnelle dans le champ de la radiothérapie et cancer du sein.

En vous souhaitant une bonne lecture.

\section{Références}

1. Bataille B, Boulanger L, Kotecki N, et al (2017) Risque de récidive locale en fonction de la classification moléculaire, exemple du cancer du sein. Oncologie 19:85-91

2. Joyon N, Penault-Llorca F, Lacroix-Triki M (2017) Classification et signatures moléculaires des cancers du sein en 2017. Oncologie 19:64-70

3. Alix-Panabières C, Pierga JY (2017) Biopsie liquide : cellules tumorales circulantes et radiothérapie. Oncologie 19:71-76

4. Bailleul-Dubois J, Bidan N, Le Bourhis X, Lagadec C (2017) Effet de la radiothérapie sur les cellules souches cancéreuses de cancer du sein : résistance, reprogrammation et traitements. Oncologie 19:77-84 